\title{
Pulsatile and Steady-State Pressure Trends in Children: A Window into the Future?
}

\author{
Justin P. Zachariah Gabriela Kovacikova \\ Department of Cardiology, Boston Children's Hospital, and Department of Pediatrics, \\ Harvard Medical School, Boston, Mass., USA
}

\section{Key Words}

Pediatric obesity · Cardiovascular risk factors · Blood pressure · Arterial stiffness .

Atherosclerosis

\begin{abstract}
The aorta has limited ability to accommodate increasing body size by remodeling. The dramatic rise in pediatric obesity threatens to overwhelm this intrinsic remodeling program and lead to abnormal aortic function. As hypothesized, pulse pressure, as an index of aortic function, has indeed risen dramatically in parallel with the rise of pediatric obesity, while at the same time mean arterial pressure, as an index of small resistance artery function, has fallen. These divergent large-artery-versus-small-artery indices may combine to explain the counterintuitive decrease in systolic blood pressure in children and adults during the global obesity pandemic. The pathophysiologic mechanisms underpinning these contrasting trends are not yet known.

(C) 2015 S. Karger AG, Basel
\end{abstract}

Across the globe, pediatric obesity rates are climbing. A concurrent rise in the prevalence of diabetes mellitus, cholesterol disorder, and hypertension can also be observed, effectively increasing childhood risk of overweight and obesity-related cardiovascular disease (CVD). In the United States, more than one third of children carry excess weight, close to $20 \%$ present with abnormal lipid levels, and almost 5\% have abnormal blood pressure (BP) [1-3]. Furthermore, it has been shown that a large proportion of children carry their CVD risk factors into adulthood; $\geq 70 \%$ of overweight children will be overweight or will become obese as adults $[4,5]$. Pediatric excess weight threatens to disturb the decreasing trend of adult coronary heart disease mortality seen over the past 30 years [6], which, if realized, would be followed by a consequent decrease in the quality of life, shorter life spans, and heavy utili- 
zation of medical resources. Furthermore, excess weight substantially increases the likelihood of high BP, the combination of which has the potential to threaten a child's cardiac health far into the future $[2,5,7-11]$.

The effects of abnormal adolescent BP on future cardiac health have been demonstrated in recent longitudinal studies. For example, systolic (SBP) and diastolic BP (DBP) were measured in Swedish adolescent male military conscripts and were found to predict future CVD mortality in a continuous fashion [12]. Furthermore, an inverse-graded relationship between BP category and freedom from CVD events was seen in predominantly Caucasian male college matriculates [13]. Thus, adolescent BP adds additional information and could play a role in CVD prevention. However, the association between childhood elevated BP and adult mortality has not been thoroughly investigated. One study by Franks et al. [14] reported a positive association between physician-reported hypertension and early mortality in children of Native American origin (mean age 11.3 years). Importantly, it was found that CVD was not the primary mechanism that led to early mortality in this population despite an inordinately high prevalence of CVD risk factors. Secondly, a recent temporal study drawn from administrative databases revealed that the incidence of stroke decreased in all groups with the exception of adolescents and young adults, where it increased dramatically. In these children, the prevalence of CVD risk factors was greater [15]. Therefore, early-life BP does appear to predict life-threatening events both in the short and long term, although its role in younger children is unclear.

The challenges inherent in demonstrating the association between childhood BP and cardiac events are multifarious and complex. First, longitudinal tracking is logistically challenging. Extensive resources, dedicated funding, and institutional memory all determine an institution's success in collecting statistically and clinically significant data on CVD events over a time span of 30-50 years. Based on the current scientific funding climate, it is debatable whether or not long-term studies can be carried out.

The second challenge involves the ever-changing nature of kids. If children are constantly changing, how do we distinguish between natural variation and accumulated CVD insults? Among adults, data suggests that subclinical atherosclerosis can predict CVD events. The role of carotid intima-media thickness (CIMT) among clinical and subclinical atherosclerosis patients is intriguing. Studies have shown that common CIMT does not offer more information than CVD risk factors alone, but that internal carotid plaque adds to discrimination in comparison to classic CVD risk factors [16]. While adult physicians may not find common CIMT valuable in its predictive power, CIMT may prove to be an advantageous tool for pediatric-focused investigators searching for a summary index of accumulated and fluctuating CVD risk factors. More data is needed in order to fully understand the effect of early-life CIMT on future CVD events.

A third challenge is regarding what constitutes gold-standard data. Leaning on principles outlined by Hill's criteria on causation and supported by research that shows how intuitively satisfying cardiac interventions can actually be misleading or dangerous, the scientific community is beginning to recognize that the 'right' answer is sometimes irrational $[17,18]$. In an effort to avoid such miscalculations, randomized blinded placebo-controlled trials (RCT) have been developed in order to determine the 'truth'. The RCT undoubtedly bolsters scientific findings, but does a lack of RCT then condone inaction or prevent and dissuade alternative action? For example, does one need an RCT to determine whether children should eat vegetables? These types of questions exemplify the difficulties in testing primordial and primary CVD prevention strategies.

In the absence of an RCT conducted over several decades, several recent observational studies provide useful data. Long-term observational studies have revealed that single pointin-time measurements of CVD risk factors in youth can predict subclinical atherosclerotic 
changes, including CIMT and arterial stiffness, especially among the adolescent population. For example, $41 \%$ of children with normal BP presented with elevated BP as adults, while $59 \%$ of children with elevated BP maintained their elevated status in adulthood $[19,20]$. In another study [21], researchers investigated the association between childhood BP and left ventricular (LV) hypertrophy, a precursor to congestive heart failure. Just as middle-aged BP predicts later LV mass and early adult BP predicts middle-aged LV mass, recent studies have demonstrated that elevated childhood BP and BMI independently determine early-adult LV mass [21, 22]. Moreover, a recent observational study [20] has demonstrated that children who improved their BP upon entering adulthood had healthier CIMT compared to those with persistently elevated BP. The physiology of the improvement in this study may relate to a decreasing BMI in the resolution group.

In order to mitigate CVD risk factors in patients, providers and researchers are tempted to simply recommend weight loss. However, the answer is not that simple. While nearly 1 in 20 children has elevated BP and excess weight doubles or triples the risk of abnormal BP, it turns out that population data from the US reveals approximately $50 \%$ of children with abnormal BP are of normal weight [23]. This counterintuitive outcome can be derived from a simple calculation: a small proportion of a large number still amounts to a relatively larger number. Applied here, a relatively smaller proportion of a larger normal-weight cohort, in comparison to a large proportion of the smaller obese population, still amounts to a significant number of normal-weight youth with abnormal BP.

Trends in population BP over the past three decades are also counterintuitive. Reliable data illustrates a pronounced increase in adult and pediatric obesity around the globe, while also showing that excess weight markedly increases the risk of hypertension in youth and adult populations [24-26]. Since pediatric excess weight and hypertension track into adulthood, it follows that we should be able to classify pediatric hypertension and excess weight as risk factors for adult hypertension and CVD [4, 27]. However, high-quality data reports a stable or decreasing SBP temporal trend in child and adult populations despite increasing obesity [28-30].

We investigated this contrary set of trends by focusing on mechanistic decomposition. Instead of analyzing BP through SBP and DBP values, it has been shown that decomposition into pulse pressure (PP; SBP - DBP) as well as mean arterial pressure (MAP; DBP + PP/3) can offer further insight into the pathophysiology of high $\mathrm{BP}$, and can also equally predict incident CVD events [31,32]. Large artery stiffness and flow pulsatility are determinants of PP, and high PP is known to predict incident CVD and is also a precursor of isolated systolic hypertension [32-36]. Key drivers of MAP include small resistance artery function and cardiac output [32]. Sorof et al. [37] found that isolated systolic elevation presented as the most common form of elevated BP in children and was more characteristic in obese children. Additional weight in children may cause incongruity between cardiac output and peripheral resistance as well as between pulsatile blood flow and aortic size. For this reason, we hypothesized that a temporal increase in PP and MAP would accompany the observed rise in pediatric excess weight. We examined the US national population-representative National Health and Nutrition Examination Surveys (NHANES) to interrogate this hypothesis.

In accordance with our postulate that excess weight may alter aortic function during childhood, data from the past three decades reveals that PP values have notably increased alongside the growing prevalence of child hood obesity. More obesity accounts for a substantial proportion of the increasing temporal trend in PP. These observations suggest either that common mechanisms are responsible for a parallel increase in both obesity and PP, or that the national increase in excess weight contributed to the increase in PP. We found that PP was related to age and height. Obesity further augmented the relation between height and PP. Obesity also amplified the relation among PP and age in males, yet not in females. Recent 
studies have shown that high PP in normotensive individuals is predictive of future hypertension, and thus the parallel increase in obesity and PP we observed foreshadows the mechanism by which the pediatric obesity epidemic may burden systolic hypertension rates through higher PP in the future [38].

Pathophysiological mechanisms that lead to high PP are exacerbated by excess weight. After the first years of life, the aorta is known to remodel in order to accommodate somatic growth. For example, large arteries increase their diameter to accommodate for higher flow [39-41]. A wider diameter decreases impedance to pulsatile flow and can actually help keep PP in a physiologic range; however, a large diameter can also increase pulsatile and mean tension on the aortic wall. Once active elastic fiber production ceases and is essentially fixed in the first couple of years of life, continuing aortic dilation increases load on elastin and hence requires extracellular matrix remodeling to transfer load from elastin to much stiffer collagen $[39,42,43]$. An increase in wall tension can lead to elastin fragmentation and deposition of stiffer matrix elements, the effects of which can accumulate and lead to increased wall stiffness [39]. In addition, children who are obese exhibit large stroke volume, increased blood volume, and higher pulsatile and steady flow, which, when paired with increased wall stiffness, can contribute to the development of high PP [44]. Thus, higher PP in obese children likely represents the interaction between greater aortic wall stiffness and higher flow, which together exceed the capacity for outward aortic remodeling [45-47]. Repeated cycles of high PP lead to more arterial stiffness with central adiposity as a key driver [35, 36].

Furthermore, the fact that the relationship between PP and height is being amplified by obesity suggests that a prominent increase in pulsatile flow in conjunction with rapid somatic growth and excess weight could overwhelm the aorta's remodeling capacity, resulting in increased PP. Alternatively, matching the aortic diameter and flow could be complicated by obesity, either due to increased aortic wall stiffness or an adverse effect on the endothelial function that inhibits adaptive remodeling through limited transduction of the flow stimulus.

The trends in MAP are complex. Although associations between higher BMI and higher MAP are expected and were found in our investigation, the average MAP decreased throughout the study period. For this reason, obesity cannot be responsible for the decreasing MAP trend. In both sexes, obesity diminished the relationship between MAP and a child's age or height. In theory, it is plausible that countervailing trends in small artery resistance versus pressure pulsatility could occur, given the fact that developing children are able to dissipate excess pulsatile energy while in a physiologic growth period through an increase in the crosssectional arterial area. For example in adults, angiogenic growth factors relate to pulsatile and steady-state parameters [48]. According to our analysis, population temporal trends in smoking do not explain the downward trend in MAP $[49,50]$. The root of the MAP decrement is unclear.

Based on our analyses of pediatric PP trends today, we can predict that a rise in pediatric PP trends today may translate into an increased risk of hypertension and exacerbated CVD endpoints in the future, especially in light of the fact that higher PP correlates with excess risk at any given SBP in adulthood. The cohorts of children examined in previous NHANES studies may reflect this prediction, as they are already starting to experience adverse consequences of elevated PP. For example, a 19\% prevalence of elevated BP has been reported in recent studies of 25- to 34-year-old subjects [51]. More troubling, given that the incident stroke risk in adults is greater in individuals with elevated PP [33,34,52], studies report a temporal increase in stroke hospitalization for persons between the age of 15 and 34 years, more of whom suffer from hypertension [15]. It is important to note that previous publications, which reported a minimized association between PP and CVD risk, may not be relevant to children and adolescents today, since the effects of perpetual exposure to increased obesity-related PP throughout somatic growth have not yet been studied [32, 52]. 
Zachariah and Kovacikova: Pulsatile and Steady-State Pressure Trends in Children: A Window into the Future?

Between 1976 and 2008, PP increased synchronously with rising rates of obesity. Therefore, it is critical that population-wide obesity is reconciled, and that mechanistic links between obesity and aortic function are elucidated. Understanding these mechanisms is the only way through which potentially severe adverse sequelae that stem from elevated pressure pulsatility, such as hypertension and consequent CVD, can be prevented.

\section{Acknowledgment}

This work was supported by the National Heart, Lung and Blood Institute Career Development Award (K23) HL 111335.

\section{Disclosure Statement}

The authors declare that they have no financial disclosures. No funding source had any influence over any part of the formulation or submission of this paper.

\section{References}

1 Kit BK, et al: Trends in serum lipids among US youths aged 6 to 19 years, 1988-2010. JAMA 2012;308:591600.

-2 Ostchega Y, et al: Trends of elevated blood pressure among children and adolescents: data from the National Health and Nutrition Examination Survey 1988-2006. Am J Hypertens 2009;22:59-67.

-3 Ogden CL, et al: Prevalence of obesity and trends in body mass index among US children and adolescents, 1999-2010. JAMA 2012;307:483-490.

4 Deshmukh-Taskar, $\mathrm{P}$, et al: Tracking of overweight status from childhood to young adulthood: the Bogalusa Heart Study. Eur J Clin Nutr 2006;60:48-57.

$\rightarrow 5$ Juhola,J, et al: Tracking of serum lipid levels, blood pressure, and body mass index from childhood to adulthood: the Cardiovascular Risk in Young Finns Study. J Pediatr 2011;159:584-590.

6 Ford ES, et al: Explaining the decrease in U.S. deaths from coronary disease, 1980-2000. N Engl J Med 2007; 356:2388-2398.

7 DiPietro L, Mossberg HO, Stunkard AJ: A 40-year history of overweight children in Stockholm: life-time overweight, morbidity, and mortality. Int J Obes Relat Metab Disord 1994;18:585-590.

-8 Freedman DS, et al: Relationship of childhood obesity to coronary heart disease risk factors in adulthood: the Bogalusa Heart Study. Pediatrics 2001;108:712-718.

$\checkmark 9$ Gunnell DJ, et al: Childhood obesity and adult cardiovascular mortality: a 57-y follow-up study based on the Boyd Orr cohort. Am J Clin Nutr 1998;67:1111-1118.

10 Juhola J, et al: Childhood physical, environmental, and genetic predictors of adult hypertension: the cardiovascular risk in young Finns study. Circulation 2012;126:402-409.

11 Juonala M, et al: Childhood adiposity, adult adiposity, and cardiovascular risk factors. N Engl J Med 2011;365: 1876-1885.

12 Sundstrom J, et al: Association of blood pressure in late adolescence with subsequent mortality: cohort study of Swedish male conscripts. BMJ 2011;342:d643.

13 Gray L, et al: Blood pressure in early adulthood, hypertension in middle age, and future cardiovascular disease mortality: HAHS (Harvard Alumni Health Study). J Am Coll Cardiol 2011;58:2396-2403.

14 Franks PW, et al: Childhood obesity, other cardiovascular risk factors, and premature death. N Engl J Med 2010;362:485-493.

15 George MG, et al: Trends in stroke hospitalizations and associated risk factors among children and young adults, 1995-2008. Ann Neurol 2011;70:713-721.

16 Polak JF, et al: Carotid-wall intima-media thickness and cardiovascular events. N Engl J Med 2011;365:213-221.

17 Echt DS, et al: Mortality and morbidity in patients receiving encainide, flecainide, or placebo. The Cardiac Arrhythmia Suppression Trial. N Engl J Med 1991;324:781-788.

18 Barter PJ, et al: Effects of torcetrapib in patients at high risk for coronary events. N Engl J Med 2007;357: 2109-2122.

19 Aatola H, et al: Lifetime risk factors and arterial pulse wave velocity in adulthood: the cardiovascular risk in young Finns study. Hypertension 2010;55:806-811.

20 Juhola J, et al: Combined effects of child and adult elevated blood pressure on subclinical atherosclerosis: the International Childhood Cardiovascular Cohort Consortium. Circulation 2013;128:217-224. 
Zachariah and Kovacikova: Pulsatile and Steady-State Pressure Trends in Children: A Window into the Future?

21 Lai CC, et al: Impact of long-term burden of excessive adiposity and elevated blood pressure from childhood on adulthood left ventricular remodeling patterns: the Bogalusa Heart Study. J Am Coll Cardiol 2014;64:15801587.

22 Sabo RT, et al: Serial childhood BMI and associations with adult hypertension and obesity: the Fels Longitudinal Study. Obesity (Silver Spring) 2012;20:1741-1743.

23 May AL, Kuklina EV, Yoon PW: Prevalence of cardiovascular disease risk factors among US adolescents, 19992008. Pediatrics 2012;12:1035-1041.

24 Must A, et al: Long-term morbidity and mortality of overweight adolescents. A follow-up of the Harvard Growth Study of 1922 to 1935. N Engl J Med 1992;327:1350-1355.

25 Sorof JM, et al: Overweight, ethnicity, and the prevalence of hypertension in school-aged children. Pediatrics 2004;113:475-482.

26 Chirinos JA, et al: Body mass index and hypertension hemodynamic subtypes in the adult US population. Arch Intern Med 2009;169:580-586.

27 Chen X, Wang Y: Tracking of blood pressure from childhood to adulthood: a systematic review and metaregression analysis. Circulation 2008;117:3171-3180.

-28 Danaei G, et al: National, regional, and global trends in systolic blood pressure since 1980: systematic analysis of health examination surveys and epidemiological studies with 786 country-years and 5.4 million participants. Lancet 2011;377:568-577.

-29 Finucane MM, et al: National, regional, and global trends in body-mass index since 1980: systematic analysis of health examination surveys and epidemiological studies with 960 country-years and 9.1 million participants. Lancet 2011;377:557-567.

-30 Din-Dzietham R, et al: High blood pressure trends in children and adolescents in national surveys, 1963 to 2002. Circulation 2007;116:1488-1496.

-31 Franklin SS, et al: Is pulse pressure useful in predicting risk for coronary heart disease? The Framingham heart study. Circulation 1999;100:354-360.

32 Franklin SS, et al: Single versus combined blood pressure components and risk for cardiovascular disease: the Framingham Heart Study. Circulation 2009;119:243-250.

-33 Staessen JA, et al: Risks of untreated and treated isolated systolic hypertension in the elderly: meta-analysis of outcome trials. Lancet 2000;355:865-872.

34 Mattace-Raso FU, et al: Blood pressure components and cardiovascular events in older adults: the Rotterdam study. J Am Geriatr Soc 2004;52:1538-1542.

-35 McEniery CM, et al: An analysis of prospective risk factors for aortic stiffness in men: 20-year follow-up from the Caerphilly prospective study. Hypertension 2010;56:36-43.

-36 McEniery CM, et al: Increased stroke volume and aortic stiffness contribute to isolated systolic hypertension in young adults. Hypertension 2005;46:221-226.

-37 Sorof JM, et al: Isolated systolic hypertension, obesity, and hyperkinetic hemodynamic states in children. J Pediatr 2002;140:660-666.

38 Kaess BM, et al: Aortic stiffness, blood pressure progression, and incident hypertension. JAMA 2012;308: 875-881.

39 Lakatta EG, Wang M, Najjar SS: Arterial aging and subclinical arterial disease are fundamentally intertwined at macroscopic and molecular levels. Med Clin North Am 2009;93:583-604.

40 Lam CS, et al: Aortic root remodeling over the adult life course: longitudinal data from the Framingham Heart Study. Circulation 2010;122:884-890.

41 Segers P, et al: Noninvasive (input) impedance, pulse wave velocity, and wave reflection in healthy middleaged men and women. Hypertension 2007;49:1248-1255.

-42 Schlatmann TJ, Becker AE: Histologic changes in the normal aging aorta: implications for dissecting aortic aneurysm. Am J Cardiol 1977;39:13-20.

43 Wagenseil JE, Mecham RP: Vascular extracellular matrix and arterial mechanics. Physiol Rev 2009;89:957-989.

44 Pflieger KL, et al: The effect of adiposity on children's left ventricular mass and geometry and haemodynamic responses to stress. Int J Obes Relat Metab Disord 1994;18:117-122.

45 Urbina EM, et al: Increased arterial stiffness is found in adolescents with obesity or obesity-related type 2 diabetes mellitus. J Hypertens 2010;28:1692-1698.

46 Aggoun Y, et al: Impaired endothelial and smooth muscle functions and arterial stiffness appear before puberty in obese children and are associated with elevated ambulatory blood pressure. Eur Heart J 2008;29:792-799.

47 Zebekakis PE, et al: Obesity is associated with increased arterial stiffness from adolescence until old age. J Hypertens 2005;23:1839-1846.

48 Zachariah JP, et al: Circulating vascular growth factors and central hemodynamic load in the community. Hypertension 2012;59:773-779.

49 Eaton DK, et al: Youth risk behavior surveillance - United States 2011. MMWR Surveill Summ 2012;61:1-162.

50 Hatsukami DK, et al: Effects of cigarette reduction on cardiovascular risk factors and subjective measures. Chest 2005;128:2528-2537.

51 Nguyen QC, et al: Discordance in national estimates of hypertension among young adults. Epidemiology 2011; 22:532-541.

52 Domanski M, et al: Pulse pressure and cardiovascular disease-related mortality: follow-up study of the Multiple Risk Factor Intervention Trial (MRFIT). JAMA 2002;287:2677-2683. 